\title{
An Efficient Approach for Identifying Constitutive Parameters of the Modified Oyane Ductile Fracture Criterion at High Temperature
}

\author{
Sergei Alexandrov, ${ }^{1}$ Yusof Mustafa, ${ }^{2}$ and Mohd Yazid Yahya ${ }^{3}$ \\ ${ }^{1}$ A.Yu. Ishlinskii Institute for Problems in Mechanics, Russian Academy of Sciences, 101-1 Prospect Vernadskogo, Moscow 119526, Russia \\ ${ }^{2}$ Faculty of Mechanical Engineering, Universiti Teknologi Malaysia, 81310 Skudai, Johor Darul Ta’zim, Malaysia \\ ${ }^{3}$ Centre for Composite, Universiti Teknologi Malaysia, 81310 Skudai, Johor Darul Ta’zim, Malaysia \\ Correspondence should be addressed to Sergei Alexandrov; sergei_alexandrov@spartak.ru
}

Received 13 September 2013; Revised 6 November 2013; Accepted 6 November 2013

Academic Editor: Timon Rabczuk

Copyright ( 2013 Sergei Alexandrov et al. This is an open access article distributed under the Creative Commons Attribution License, which permits unrestricted use, distribution, and reproduction in any medium, provided the original work is properly cited.

\begin{abstract}
The paper presents the theoretical part of a method for identifying constitutive parameters involved in the modified Oyane ductile fracture criterion at high temperature. Quite a general rigid viscoplastic model is adopted to describe material behavior. The ductile fracture criterion is in general path-dependent and involves stresses. Therefore, the identification of constitutive parameters of this criterion is a difficult task which usually includes experimental research and numerical simulation. The latter requires a precisely specified material model and boundary conditions. It is shown in the present paper that for a wide class of material models usually used to describe the behavior of materials at high temperatures, the criterion is significantly simplified when the site of fracture initiation is located on traction free surfaces. In particular, this reduced criterion does not involve stresses. Since there are well established experimental procedures to determine the input data for the reduced criterion, the result obtained can be considered as a theoretical basis for the efficient method for identifying constitutive parameters of the modified Oyane ductile fracture criterion at high temperature. The final expression can also be used in computational models to increase the accuracy of predictions.
\end{abstract}

\section{Introduction}

Empirical ductile fracture criteria are widely used by practically working engineers to predict the initiation of ductile fracture in metal forming processes. Reviews of such criteria for cold metal forming processes are provided in [1-3]. Most of these criteria include a path-dependent integral whose integrand contains invariants of the stress tensor. Therefore, the identification of constitutive parameters of ductile fracture criteria in general requires numerical simulation of forming processes which is impossible without a precisely specified material model and boundary conditions. These input data affect the result of numerical simulation. An alternative method of identifying constitutive parameters of two widely used cold ductile fracture criteria has been proposed in $[4,5]$. This method is applicable when the fracture initiation occurs at traction free surfaces and the final expressions are finite relations between two in-surface strains or the equivalent strain and two in-surface strains. In the case of hot forming, many experimental results indicate a sharp drop of the strain to fracture at a certain level of temperature and strain rate [6-8]. In the range of temperatures and strain rates where this drop does not occur, hot ductile fracture criteria are often accepted as modifications of cold ductile fracture criteria in which constitutive parameters depend on temperature and strain rate $[6,9-13]$. In particular, the criterion proposed in [14] for cold metal forming has been modified in $[10,13]$ to account for temperature and strain rate effects. Experimental verification of the applicability of this modified ductile fracture criterion to several materials has been provided in these papers as well. An efficient method for identifying constitutive parameters involved in the original criterion [14] has been proposed in [4]. However, this method is based on a rigid hardening material model 
which is not adequate for describing hot metal forming. In the present paper, the method [4] is extended to quite a general viscoplastic model.

\section{Constitutive Equations}

Rigid viscoplastic solids are often used to describe the behavior of material in hot metal forming processes (see, e.g., [15]). The constitutive equations of such solids are the yield condition and its associated flow rule. A widely adopted yield condition is

$$
\sigma_{\mathrm{eq}}=\sigma_{0} \Phi\left(\xi_{\mathrm{eq}}, \varepsilon_{\mathrm{eq}}\right)
$$

where $\sigma_{0}$ is a reference stress, $\sigma_{\text {eq }}$ is the equivalent stress, $\xi_{\text {eq }}$ is the equivalent strain rate, and $\varepsilon_{\mathrm{eq}}$ is the equivalent strain. The quantities $\sigma_{\text {eq }}, \xi_{\text {eq }}$, and $\varepsilon_{\text {eq }}$ are defined by

$$
\sigma_{\mathrm{eq}}=\sqrt{\frac{3}{2} \sqrt{\tau_{i j} \tau_{i j}}}, \quad \xi_{\mathrm{eq}}=\sqrt{\frac{2}{3}} \sqrt{\xi_{i j} \xi_{i j}}, \quad \frac{d \varepsilon_{\mathrm{eq}}}{d t}=\xi_{\mathrm{eq}},
$$

where $\sigma_{i j}$ are the components of the stress tensor, $\xi_{i j}$ are the components of the strain rate tensor, $t$ is the time, $\tau_{i j}=$ $\sigma_{i j}-\sigma \delta_{i j}$ are the components of the stress deviator tensor, $\sigma=\left(\sigma_{i j} \delta_{i j}\right) / 3$ is the hydrostatic stress, and $\delta_{i j}$ is Kronecker's symbol. With no loss of generality it is possible to assume that $\Phi(0,0)=1$. Then, $\sigma_{0}$ is the yield stress in uniaxial tension at $\xi_{\text {eq }}=0$ and $\varepsilon_{\text {eq }}=0$. The flow rule associated with the yield condition (1) results in

$$
\xi_{i j}=\lambda \tau_{i j}
$$

where $\lambda$ is a nonnegative multiplier. Using (1) this multiplier can be eliminated in (3) to give

$$
\xi_{i j}=\frac{3}{2} \frac{\xi_{\mathrm{eq}} \tau_{i j}}{\sigma_{0} \Phi\left(\xi_{\mathrm{eq}}, \varepsilon_{\mathrm{eq}}\right)} .
$$

The ductile fracture criterion applicable at high temperatures and strain rates is [13]

$$
\int_{0}^{t_{f}}\left(1+\frac{\sigma}{B \sigma_{\mathrm{eq}}}\right) \xi_{\mathrm{eq}} d t=C\left(\xi_{\mathrm{eq}}, T\right)
$$

where $t$ is the time, $t_{f}$ is the value of $t$ at the instant of fracture initiation, and $B$ is a material constant. $C$ depends on the equivalent strain rate and temperature, $T$. The ductile fracture criterion (5) is a modification of the criterion proposed in [14] for cold metal forming processes. It is worthwhile of noting that the model chosen demands that the principal axes of the stress and strain rate tensors coincide.

\section{Free Surface Fracture}

Assume that fracture initiates at a point of a traction free surface. It is evident that one of the principal stress directions is orthogonal to this surface. Therefore, one of the principal strain rate directions is also orthogonal to this surface and an infinitesimal material fiber coinciding with this principal direction at the initial instant is fixed in the material. Let $\xi_{1}$ be the principal strain rate associated with this direction. In this special case

$$
\varepsilon_{1}=\int_{0}^{t} \xi_{1} d t
$$

where $\varepsilon_{1}$ is the principal logarithmic strain. Equation (6) is valid at any large strain. The principal stress orthogonal to the free surface vanishes; $\sigma_{1}=0$. It follows from this equation that

$$
\sigma=-\tau_{1}
$$

at the free surface. Substituting (7) into (5) and using (1) give

$$
\int_{0}^{t_{f}}\left[1-\frac{\tau_{1}}{B \sigma_{0} \Phi\left(\xi_{\mathrm{eq}}, \varepsilon_{\mathrm{eq}}\right)}\right] \xi_{\mathrm{eq}} d t=C\left(\xi_{\mathrm{eq}}, T\right) .
$$

It follows from (4) that

$$
\frac{2}{3} \xi_{1}=\frac{\xi_{\mathrm{eq}} \tau_{1}}{\sigma_{0} \Phi\left(\xi_{\mathrm{eq}}, \varepsilon_{\mathrm{eq}}\right)}
$$

Replacing the integrands in (8) by means of (9) leads to

$$
\int_{0}^{t_{f}}\left[\xi_{\mathrm{eq}}-\frac{2}{3} \frac{\xi_{1}}{B}\right] d t=C\left(\xi_{\mathrm{eq}}, T\right) .
$$

Using (2) and (6) integration in this equation can be carried out analytically to give

$$
\varepsilon_{\mathrm{eq}}^{f}-\frac{2}{3} \frac{\varepsilon_{1}^{f}}{B}=C\left(\xi_{\mathrm{eq}}, T\right),
$$

where $\varepsilon_{\mathrm{eq}}^{f}$ and $\varepsilon_{1}^{f}$ are the values of $\varepsilon_{\mathrm{eq}}$ and $\varepsilon_{1}$, respectively, at the instant of fracture initiation. Let $\varepsilon_{2}$ and $\varepsilon_{3}$ be the in-surface principal logarithmic strains and let $\varepsilon_{2}^{f}$ and $\varepsilon_{3}^{f}$ be their values at the instant of fracture initiation, respectively. It follows from the incompressibility equation that $\varepsilon_{3}^{f}+\varepsilon_{2}^{f}+\varepsilon_{1}^{f}=0$. Using this equation, $\varepsilon_{1}^{f}$ can be eliminated in (11) to give

$$
\varepsilon_{\mathrm{eq}}^{f}+\frac{2}{3} \frac{\left(\varepsilon_{2}^{f}+\varepsilon_{3}^{f}\right)}{B}=C\left(\xi_{\mathrm{eq}}, T\right)
$$

This relation is the fracture criterion at the free surface. Its remarkable property is that the left hand side of (12) is independent of stress components. The in-surface finite strains at the instant of fracture initiation can be found using high speed photography [12]. The equivalent strain is in general path-dependent. Therefore, the strain path should be determined to calculate $\varepsilon_{\mathrm{eq}}^{f}$. However, since the material is supposed to be incompressible, it is sufficient to find the dependence of $\varepsilon_{2}$ on $\varepsilon_{3}$ [16]. This dependence can be found using high speed photography. 


\section{Conclusions}

The main result of the present paper is that the ductile fracture criterion (5) involving stresses reduces to (12) when the initiation of fracture occurs at a point of a traction free surface. Equation (12) is simply a relation between the equivalent strain and two in-surface strains. Therefore, there is no need to find experimentally and numerically the state of stress at the site of fracture initiation. On the contrary, (12) can be used in numerical codes. This should increase the accuracy of predictions because there is no need to calculate the state of stress at the site of fracture initiation. As an example, it is instructive to apply (12) to theoretical/experimental results obtained in [17]. In this work the finite element method and compression of cylindrical specimens of Ti40 alloy were used to identify the constitutive parameters of the fracture criterion (5) at $B=2 / 3$. As a result, it has been found (in our nomenclature) that

$$
\int_{0}^{t_{f}}\left(1+\frac{3 \sigma}{2 \sigma_{\mathrm{eq}}}\right) \xi_{\mathrm{eq}} d t=2.227-0.0493 \ln Z
$$

Here $Z$ is the Zener-Hollomon parameter which is a temperature compensated strain rate. Using (12), it is possible to control the accuracy of (13) by means of simpler methods than those applied in [17]. In particular, (13) takes the following form:

$$
\varepsilon_{\mathrm{eq}}^{f}+\varepsilon_{2}^{f}+\varepsilon_{3}^{f}=2.227-0.0493 \ln Z .
$$

According to experimental results presented in [17] the equivalent strain to fracture is a linear function of $\ln Z$. These data are in agreement with (14) if and only if strain paths in the experiment are linear or, at least, close enough to linear. Of course, this condition should be verified by experiment. However, it is worthwhile of noting that strain paths in cold upsetting are usually convex down [18-20].

Equation (12) has been derived for quite a general viscoplastic model. Therefore, there is no need to specify the model to apply this equation in conjunction with experimental data. Finally, boundary conditions have no effect on this equation. Obviously, some boundary conditions such as a friction boundary condition have a great effect on the initiation of ductile fracture. However, all these possible effects are automatically taken into account in the measured values of the in-surface principal strains. To summarize, it is believed that the aforementioned mathematical properties of the ductile fracture criterion considered provide a theoretical basis for an efficient theoretical/experimental method for the identification of constitutive parameters of the ductile fracture criterion [13] at high temperature.

The general method of analysis used in the present paper has been also adopted in [21] to reduce the modified Cockcroft-Latham ductile fracture criterion to a simple form not involving stresses. However, the basic assumptions and final results are quite different. In particular, in the case of the modified Cockcroft-Latham ductile fracture criterion the general method is applicable if and only if the site of fracture initiation coincides with the point of intersection of two planes of symmetry and a traction free surface. On the other hand, (12) is valid at any point of a traction free surface. This difference may affect the choice of specimens for experiment. Moreover, (12) involves the equivalent strain which is a pathdependent integral whereas the final expression in [18] is a relation between two finite in-surface strains at the instant of fracture initiation. This difference may affect the choice of experimental techniques to measure kinematic variables.

The main assumption to derive (12) from the fracture criterion (5) is that the site of fracture initiation is located at a traction free surface. This condition is satisfied in many sheet forming processes. Therefore, the simplified ductile fracture criterion in the form of (12) can be directly adopted to predict the initiation of ductile fracture in such processes.

\section{Acknowledgments}

The research described in this paper has been supported by the Grants RFBR-13-08-00969 and NSH-3842.2012.1.

\section{References}

[1] A. G. Atkins, "Fracture in forming," Journal of Materials Processing Technology, vol. 56, no. 1-4, pp. 609-618, 1996.

[2] M. A. Shabara, A. A. El-Domiaty, and A. Kandil, "Validity assessment of ductile fracture criteria in cold forming," Journal of Materials Engineering and Performance, vol. 5, no. 4, pp. 478488, 1996.

[3] R. Hambli and M. Reszka, "Fracture criteria identification using an inverse technique method and blanking experiment," International Journal of Mechanical Sciences, vol. 44, no. 7, pp. 13491361, 2002.

[4] D. Vilotić, M. Plančk, S. Grbić, S. Alexandrov, and N. Chikanova, "An approach to determining the workability diagram based on upsetting tests," Fatigue and Fracture of Engineering Materials and Structures, vol. 26, no. 4, pp. 305-310, 2003.

[5] S. Alexandrov and D. Vilotic, "A theoretical-experimental method for the identification of the modified Cockroft-Latham ductile fracture criterion," Proceedings of the Institution of Mechanical Engineers C, vol. 222, no. 9, pp. 1869-1872, 2008.

[6] S. Alexandrov, P. T. Wang, and R. E. Roadman, "A fracture criterion of aluminum alloys in hot metal forming," Journal of Materials Processing Technology, vol. 160, no. 2, pp. 257-265, 2005.

[7] O. Sabokpa, A. Zarei-Hanzaki, and H. R. Abedi, "An investigation into the hot ductility behavior of AZ81 magnesium alloy," Materials Science and Engineering A, vol. 550, pp. 31-38, 2012.

[8] J. Deng, Y. C. Lin, S. S. Li, J. Chen, and Y. Ding, "Hot tensile deformation and fracture behaviors of AZ31 magnesium alloy," Materials and Design, vol. 49, pp. 209-219, 2013.

[9] N. Ogawa, M. Shiomi, and K. Osakada, "Forming limit of magnesium alloy at elevated temperatures for precision forging," International Journal of Machine Tools and Manufacture, vol. 42, no. 5, pp. 607-614, 2002.

[10] X. Zhang, W. Zeng, Y. Shu, Y. Zhao, H. Yu, and Y. Zhou, "Fracture criterion of Ti40 alloy based on the Zener-Hollomon parameter at hot forming," Rare Metal Materials and Engineering, vol. 37, no. 4, pp. 604-608, 2008.

[11] J. T. Yeom, J. H. Kim, J. H. Kim, J. K. Hong, and J. S. Lee, "Hot forging design of cam for vessel engine using finite element 
analysis and ductile fracture criteria," Steel Research International, vol. 81, pp. 298-301, 2010.

[12] Y. Zhu, W. Zeng, F. Zhang, Y. Zhao, X. Zhang, and K. Wang, "A new methodology for prediction of fracture initiation in hot compression of Ti40 titanium alloy," Materials Science and Engineering A, vol. 553, pp. 112-118, 2012.

[13] J. He, Z. Cui, F. Chen, Y. Xiao, and L. Ruan, "The new ductile fracture criterion for $30 \mathrm{Cr} 2 \mathrm{Ni} 4 \mathrm{MoV}$ ultra-super-critical rotor steel at elevated temperatures," Materials and Design, vol. 52, pp. 547-555, 2013.

[14] M. Oyane, T. Sato, K. Okimoto, and S. Shima, "Criteria for ductile fracture and their applications," Journal of Mechanical Working Technology, vol. 4, no. 1, pp. 65-81, 1980.

[15] Y.-M. Guo, Y. Yokouchi, and H. Suzuki, "Analysis of hot forward-backward extrusion by the visco-plastic finite-element method," Journal of Materials Processing Technology, vol. 38, no. 1-2, pp. 103-113, 1993.

[16] S. Alexandrov, D. Vilotic, Z. Konjovic, and M. Vilotic, "An improved experimental method for determining the workability diagram," Experimantal Mechanics, vol. 53, pp. 699-711, 2013.

[17] X.-M. Zhang, W.-D. Zeng, Y. Shu et al., "Fracture criterion for predicting surface cracking of Ti40 alloy in hot forming processes," Transactions of Nonferrous Metals Society of China, vol. 19, no. 2, pp. 267-271, 2009.

[18] H. A. Kuhn, P. W. Lee, and T. Erturk, "Fracture criterion for cold forming," Journal of Engineering Materials and Technology of the ASME, vol. 95, pp. 213-218, 1973.

[19] S. I. Oh and S. Kobayashi, "Workability of aluminum alloy 7075t6 in upsetting and rolling," Journal of Engineering for IndustryTransactions of the ASME, vol. 98, no. 3, pp. 800-806, 1976.

[20] J. J. Shah and H. A. Kuhn, "An empirical formula for workability limits in cold upsetting and bolt heading," Journal of Applied Metalworking, vol. 4, no. 3, pp. 255-261, 1986.

[21] S. Alexandrov and Y. R. Jeng, "An efficient method for the identification of the modified Cockroft-Latham fracture criterion at elevated temperature," Archive of Applied Mechanics, vol. 83, no. 12, pp. 1801-1804, 2013. 


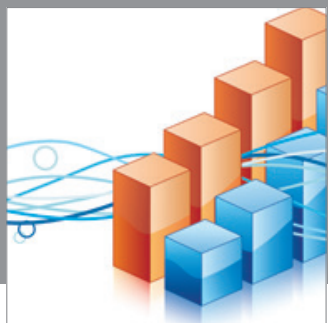

Advances in

Operations Research

mansans

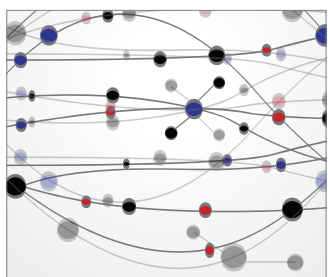

The Scientific World Journal
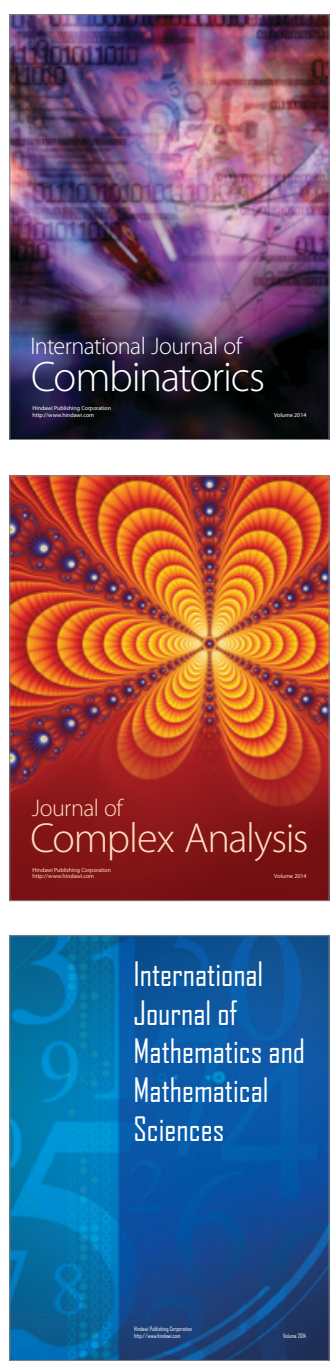
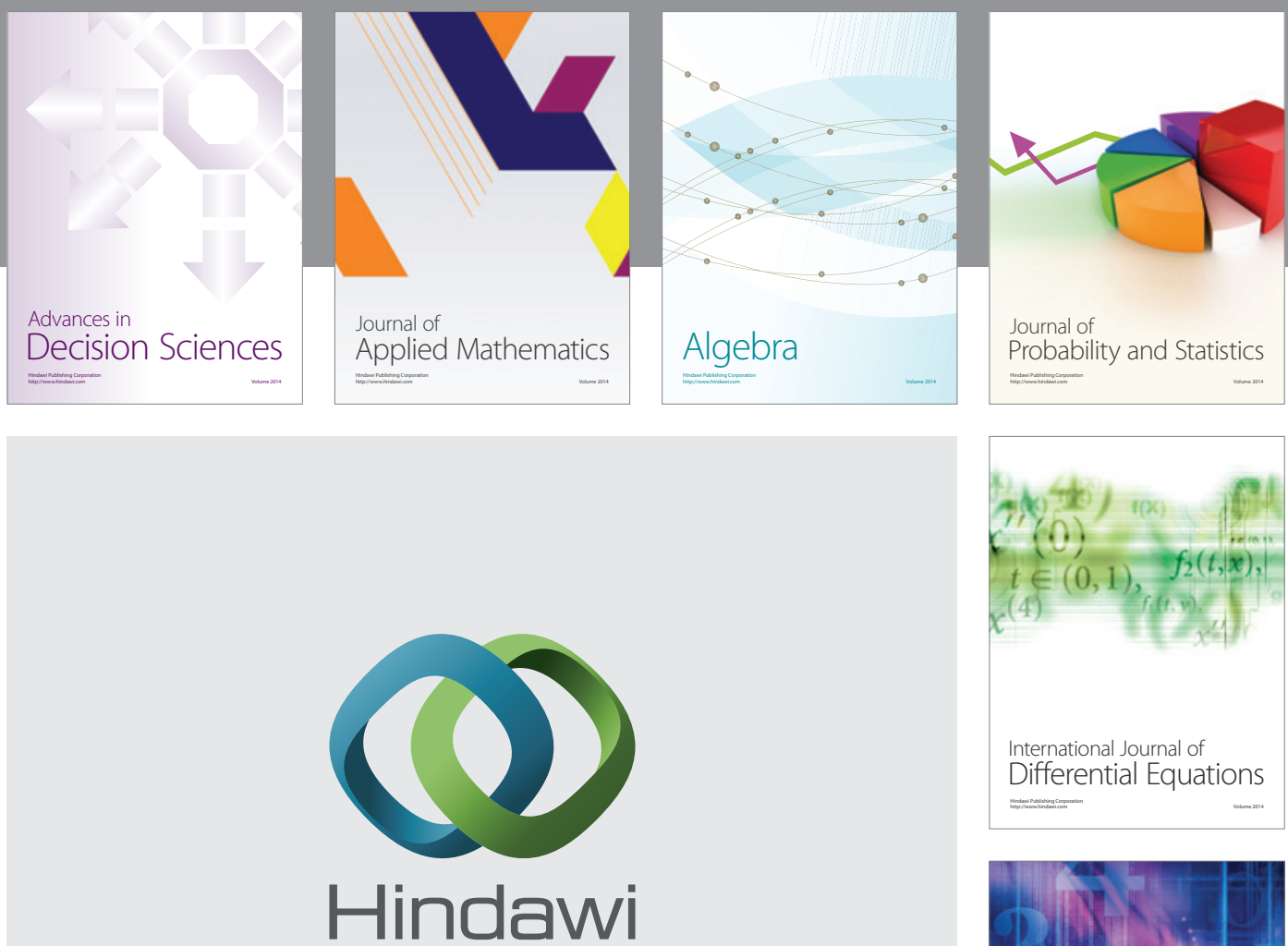

Submit your manuscripts at http://www.hindawi.com
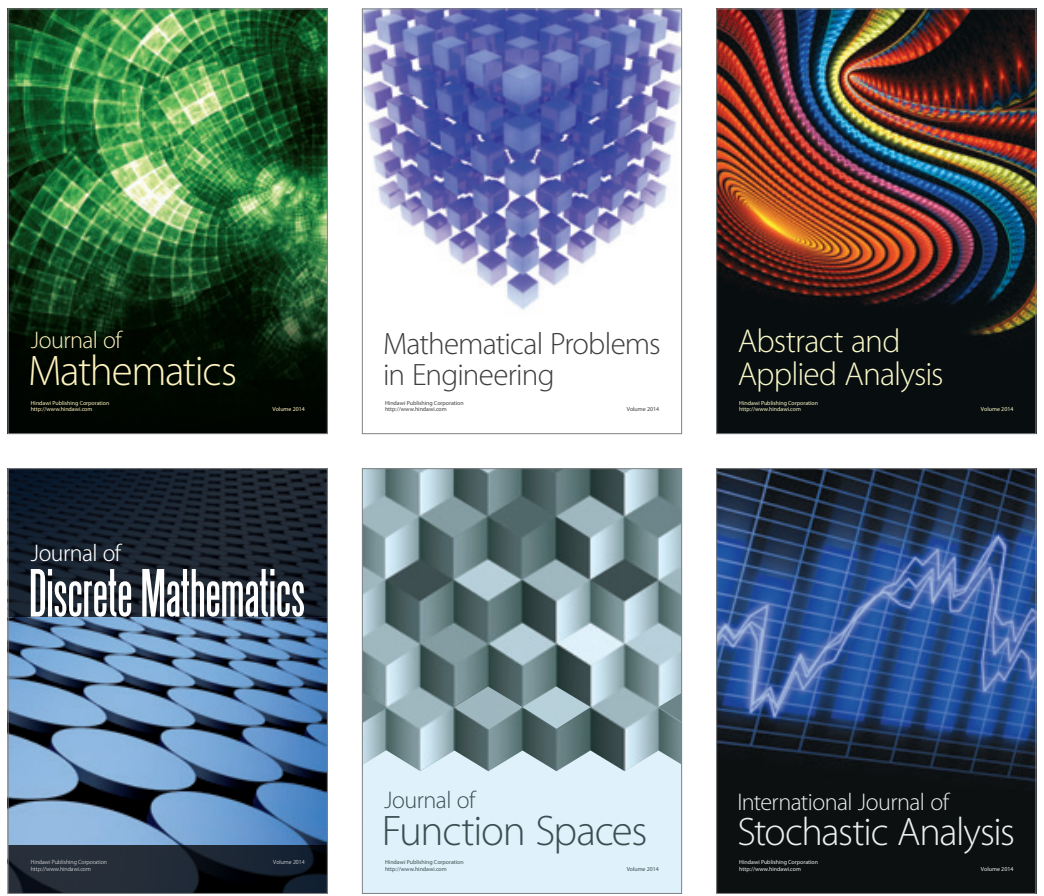

Journal of

Function Spaces

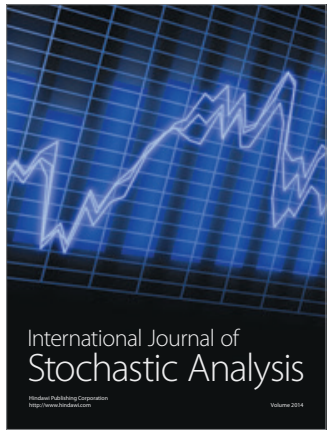

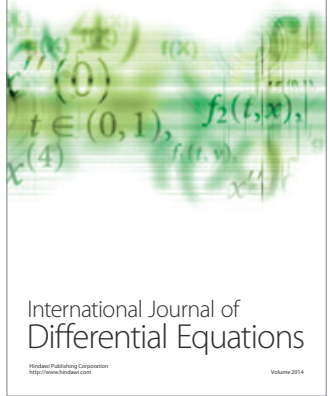
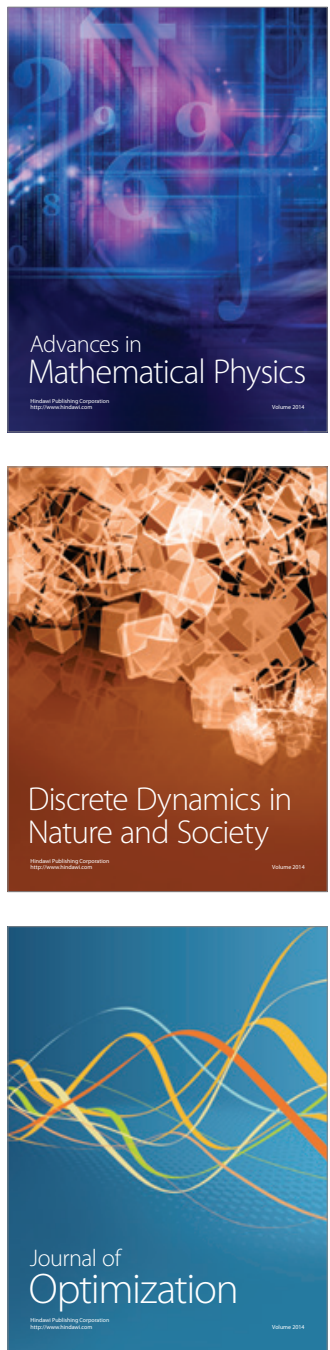